\title{
Capacity of the Discrete Memoryless Energy Harvesting Channel with Side Information
}

\author{
Omur Ozel $^{1}$, Kaya Tutuncuoglu ${ }^{2}$, Sennur Ulukus ${ }^{1}$, and Aylin Yener ${ }^{2}$ \\ ${ }^{1}$ Department of Electrical and Computer Engineering, University of Maryland, College Park, MD 20742 \\ ${ }^{2}$ Department of Electrical Engineering, The Pennsylvania State University, University Park, PA 16802
}

\begin{abstract}
We determine the capacity of a discrete memoryless communication channel with an energy harvesting transmitter and its battery state information available at the transmitter and the receiver. This capacity is an upper bound for the problem where side information is available only at the transmitter. Since channel output feedback does not increase the capacity in this case, we equivalently study the resulting finite-state Markov channel with feedback. We express the capacity in terms of directed information. Additionally, we provide sufficient conditions under which the capacity expression is further simplified to include the stationary distribution of the battery state. We also obtain a single-letter expression for the capacity with battery state information at both sides and an infinite-sized battery. Lastly, we consider achievable schemes when side information is available only at the transmitter for the case of an arbitrary finite-sized battery. We numerically evaluate the capacity and achievable rates with and without receiver side information.
\end{abstract}

\section{INTRODUCTION}

We consider a communication channel with an energy harvesting transmitter and its battery state information available at the transmitter and the receiver as shown in Fig. 1. In this channel, energy needed for communication is harvested by the transmitter throughout the communication, and the energy of each transmitted code symbol is constrained to the available battery energy in that channel use. The capacity of this channel with only transmitter side battery state information has been determined in recent work for two extreme cases in the Gaussian setting: When the battery size is unlimited, [1] showed that the capacity is equal to the capacity of the same system with an average power constraint equal to the average recharge rate. When the battery size is zero, [2] showed that the capacity is achieved by using Shannon strategies [3]. In this paper, we determine the capacity of this channel for a discrete memoryless setting with an arbitrary finite battery size, when battery state information is available at both sides. This constitutes an upper bound for the capacity when battery state information is available only at the transmitter.

A communication channel with an energy harvesting transmitter is an instance of a state-dependent channel with input dependence and memory in the state process. We model energy arrivals as multiples of a fixed quanta, and obtain a physical layer which has a discrete alphabet based on this quanta. Consequently, we obtain a finite-state Markov channel where the

This work was supported by NSF Grants CNS 09-64364 and CNS 0964632.

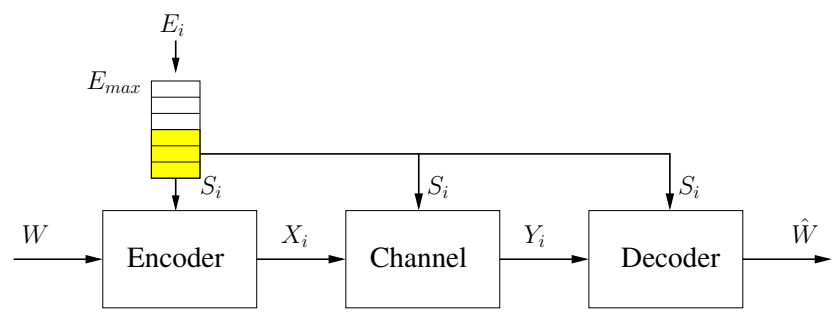

Fig. 1. The channel with an energy harvesting transmitter with a finite-sized battery. The battery state information is available at both sides.

state process interacts with the channel input. We determine the capacity of this finite-state Markov channel when battery state information is available at both the transmitter and the receiver. Since the battery state information is available at the receiver, the information flows through both the physical channel and the battery. That is, the channel uncertainty in this case is due both to the error the physical channel introduces and to the uncertainty in the energy arrival process. In view of [4], in this case, the output feedback does not increase capacity. Thus, we express the capacity as the maximum directed information between the input and the physical channel output and the battery state. Moreover, utilizing the results reported in [5], we find sufficient conditions for which the optimal input distribution is stationary and the capacity is expressed in a simpler form in terms of stationary probabilities of the battery states. We also find a single-letter capacity expression for the infinite-sized battery case in which the finite-state results in [4], [5] are no longer valid.

Next, we consider the case when battery state information is available at the transmitter only. The capacity for this case with a finite-sized battery is an open problem in general [6], [7]. In the special case of noiseless binary channel and unitsized battery, we showed in [6] that the channel is equivalent to an i.i.d. additive geometric-noise timing channel with causal information of the noise available at the transmitter. This equivalence enabled a single-letter capacity expression with an auxiliary random variable. In this paper, we observe that the approach in [6] is still suitable when battery size is larger than one, and develop an achievable scheme based on the timing channel in [6]. The key to this scheme is the fact that the additive noise in the timing channel, which is causally available to the transmitter, has memory and input dependence in a suitable form, allowing us to determine a new class of 
achievable schemes combining the method in [8] and Shannon strategies in [3]. We calculate the achievable rate by using the simulation-based method in [8]. We numerically evaluate and compare the capacity and achievable rates with and without battery state information at the receiver.

\section{The Channel Model}

We consider a channel with an energy harvesting transmitter. The battery in the transmitter can store at most $E_{\max }$ units of energy. Input symbols belong to the set $\{0,1, \ldots, K\}$. Each symbol $k$ has $k$-unit energy cost. When channel input $X_{i}$ is transmitted in the $i$ th channel use, the receiver gets $Y_{i}$. The stochastic relation $p(y \mid x)$ between the input and the output is determined by the underlying physical channel.

At each channel use, the transmitter both harvests energy and transmits a symbol. The order of harvesting and transmission in a channel use is as follows: $S_{i}$ denotes the energy available in the battery at channel use $i$. The transmitter observes the available battery energy $S_{i}$ and transmits a symbol $X_{i}$. The energy of this symbol is constrained by the battery energy: $X_{i} \leq S_{i}$. After sending the symbol, the transmitter harvests energy. Energy arrivals (harvesting) is modeled as an i.i.d. process with $E_{i} \in\{0,1, \ldots,|\mathcal{E}|\}$ and $\operatorname{Pr}\left[E_{i}=e\right]=q_{e}$ for $e \in\{0,1, \ldots,|\mathcal{E}|\}$. Incoming energy $E_{i}$ is first stored in the battery, if there is space, before it is used for transmission. Since the battery has finite size, energies may overflow and get wasted. The battery state is updated as:

$$
S_{i+1}=\min \left\{S_{i}-X_{i}+E_{i}, E_{\max }\right\}
$$

In view of (1) and the physical channel model, the battery level $S_{i}$ and the channel output $Y_{i}$ evolve according to the following joint distribution:

$$
p\left(s_{i+1}, y_{i} \mid x_{i}, s_{i}\right)=p\left(y_{i} \mid x_{i}\right) p\left(s_{i+1} \mid x_{i}, s_{i}\right)
$$

We note that $S_{i}$ is a state for this channel that is available at both the transmitter and the receiver. Therefore, information flows through both the physical channel $p(y \mid x)$ as well as the battery state. The uncertainty is introduced due to both physical channel and the energy arrival process. Note that even when the channel is noiseless, uncertainty of the battery energy at the transmitter side makes it challenging for the receiver to decode the messages of the transmitter as the state has memory and input dependence.

\section{CAPACity With BATTERy STATE INFORMATION AVAILABLE AT BOTH SIDES}

Let us define two variables $Y_{1 i} \triangleq S_{i+1}$ and $Y_{2 i} \triangleq Y_{i}$ and express the model in (2) in terms of the new definitions as:

$$
p\left(y_{1 i}, y_{2 i} \mid x_{i}, y_{1(i-1)}\right)
$$

That is, $y_{1(i-1)}$ acts as a state, which is available at the transmitter and the receiver. The model in (3) was previously studied in [4, Section VIII]. Since the channel in (3) is connected in the sense of [4, Definition 3], the channel capacity is independent of the initial state and is characterized as in the following theorem (see also [4, Appendix VIII]).

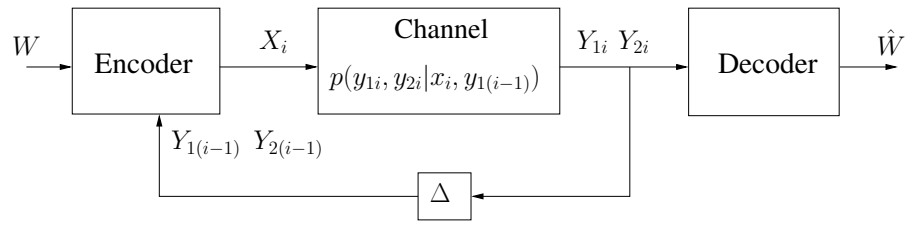

Fig. 2. Virtual channel model with feedback. Presence of the feedback of $Y_{2}$ does not affect the capacity.

Theorem 1 The channel capacity for (3) is:

$$
C=\lim _{N \rightarrow \infty} \max _{p\left(x_{i} \mid y_{1(i-1)}\right)} \frac{1}{N} \sum_{i=1}^{N} I\left(X_{i} ; Y_{1 i}, Y_{2 i} \mid Y_{1(i-1)}\right)
$$

Note that the expression in (4) is the maximum directed information from the input $X$ to the outputs $Y_{1}, Y_{2}$ for the channel in (3). Computation of (4) is possible by, e.g., the algorithm in [9], which is a combination of Blahut-Arimoto algorithm and dynamic programming.

In view of [4, Theorem 19], feedback of the channel output $Y_{2}$ does not increase the capacity (see also [10]). We note that the channel model (3) with the feedback of $Y_{2}$, as shown in Fig. 2, also matches with the model in [5]. It is shown in [5] that under some technical conditions, the capacity is achieved by stationary input distributions and it can be expressed in terms of the stationary probability of the outputs $Y_{1}$ and $Y_{2}$. Specifically, the channel transition probability must satisfy strong irreducibility and strong aperiodicity conditions in [5]. Our goal is to extend the results in [5] for the channel in (3). To this end, we first state the following lemma.

Lemma 1 Let $\left\{M_{1 i}\right\}$ and $\left\{M_{2 i}\right\}$ be strongly irreducible and strongly aperiodic Markov chains with a common input $X_{i}$. If $M_{1 i} \rightarrow X_{i} \rightarrow M_{2 i}$ holds, joint Markov process $\left\{M_{1 i}, M_{2 i}\right\}$ is also strongly irreducible and strongly aperiodic.

Proof: Since $M_{1 i} \rightarrow X_{i} \rightarrow M_{2 i}$ holds, we have:

$$
\begin{aligned}
& p\left(m_{1 i}, m_{2 i} \mid x_{i}, m_{1(i-1)}, m_{2(i-1)}\right) \\
& \quad=p\left(m_{1 i} \mid x_{i}, m_{1(i-1)}\right) p\left(m_{2 i} \mid x_{i}, m_{2(i-1)}\right)
\end{aligned}
$$

In view of (5), any path from the joint state $\left(m_{1}, m_{2}\right)$ to $\left(\tilde{m}_{1}, \tilde{m}_{2}\right)$ requires $\left\{M_{1 i}\right\}$ to travel from $m_{1}$ to $\tilde{m}_{1}$ and $\left\{M_{2 i}\right\}$ from $m_{2}$ to $\tilde{m}_{2}$, respectively. Therefore, all possible paths from $\left(m_{1}, m_{2}\right)$ to $\left(\tilde{m}_{1}, \tilde{m}_{2}\right)$ is a Cartesian product of all paths from $m_{1}$ to $\tilde{m}_{1}$ in $\left\{M_{1 i}\right\}$ and from $m_{2}$ to $\tilde{m}_{2}$ in $\left\{M_{2 i}\right\}$. Whenever $\left\{M_{1 i}\right\}$ and $\left\{M_{2 i}\right\}$ are individually strongly irreducible, there exists a path from any $m_{1}$ to $\tilde{m}_{1}$ in $\left\{M_{1 i}\right\}$ and from $m_{2}$ to $\tilde{m}_{2}$ in $\left\{M_{2 i}\right\}$ and therefore, there exits a path from any $\left(m_{1}, m_{2}\right)$ to $\left(\tilde{m}_{1}, \tilde{m}_{2}\right)$ in $\left\{M_{1 i}, M_{2 i}\right\}$, which proves that $\left\{M_{1 i}, M_{2 i}\right\}$ is also strongly irreducible. Similarly, due to the Cartesian product property, lengths of paths from any $\left(m_{1}, m_{2}\right)$ to $\left(\tilde{m}_{1}, \tilde{m}_{2}\right)$ are common multiples of lengths of paths from $m_{1}$ to $\tilde{m}_{1}$ in $\left\{M_{1 i}\right\}$ and lengths of paths from $m_{2}$ to $\tilde{m}_{2}$ in $\left\{M_{2 i}\right\}$. Therefore, all possible lengths of paths from $\left(m_{1}, m_{2}\right)$ to itself must have greatest common divisor 1 and $\left\{M_{1 i}, M_{2 i}\right\}$ is strongly aperiodic as otherwise either $\left\{M_{1 i}\right\}$ or $\left\{M_{2 i}\right\}$ is not strongly aperiodic. 
Lemma 1 states that two strongly irreducible and strongly aperiodic Markov chains driven by a single input is jointly strongly irreducible and strongly aperiodic if they are conditionally independent given the input. Note that this conditional independence is satisfied by the energy harvesting model in (2)-(3). We are now ready to prove the following theorem.

Theorem 2 Assume that the channel $p\left(y_{1 i} \mid x_{i}, y_{1(i-1)}\right)$ is strongly irreducible and strongly aperiodic and let the channel $Q_{k}=p\left(y_{1 i}, y_{2 i} \mid x_{i}, y_{1(i-1)}=k\right)$ have a rank $|\mathcal{X}|$ transition matrix for any given $y_{1(i-1)}=k$. Moreover, assume that $Y_{1 i} \rightarrow X_{i} \rightarrow Y_{2 i}$ holds with $p\left(y_{2 i} \mid x_{i}\right)>0$ for all $y_{2 i}$ and $x_{i}$. Then, the capacity of the channel in (3) is:

$$
C=\max _{p\left(x \mid \tilde{y}_{1}\right)} \sum_{k=1}^{\left|\mathcal{Y}_{1}\right|} \pi_{k} I\left(X ; Y_{1}, Y_{2} \mid \tilde{Y}_{1}=k\right)
$$

where $\tilde{Y}_{1}$ denotes the one-unit delayed feedback of $Y_{1}$.

Proof: In view of Lemma 1, the hypothesis in [5, Theorem 6] are satisfied for the channel $p\left(y_{1 i}, y_{2 i} \mid x_{i}, y_{1(i-1)}, y_{2(i-1)}\right)$. In particular, by [5, Lemma 6], the rank condition we stated in the theorem implies that the technical condition in the hypothesis in [5, Theorem 6] is satisfied. Therefore, the capacity $C$ is:

$$
\begin{aligned}
& \max _{p\left(x \mid \tilde{y}_{1}, \tilde{y}_{2}\right)} \sum_{k_{1}} \sum_{k_{2}} \pi_{k_{1}, k_{2}} I\left(X ; Y_{1}, Y_{2} \mid \tilde{Y}_{1}=k_{1}, \tilde{Y}_{2}=k_{2}\right) \\
& =\max _{p\left(x \mid \tilde{y}_{1}, \tilde{y}_{2}\right)} \sum_{k_{1}} \pi_{k_{1}} \sum_{k_{2}} \pi_{k_{2} \mid k_{1}} I\left(X ; Y_{1}, Y_{2} \mid \tilde{Y}_{1}=k_{1}\right)
\end{aligned}
$$

where (8) follows from the fact that the channel in (3) does not have dependence on $\tilde{Y}_{2}$. In (8), the input distributions are selected based on the past channel outputs, i.e., $p\left(x \mid \tilde{y}_{1}=\right.$ $\left.k_{1}, \tilde{y}_{2}=k_{2}\right)$. Now, consider the marginal distribution of $X$ given $\tilde{Y}_{1}=k_{1}$ :

$$
p\left(x \mid \tilde{y}_{1}=k_{1}\right)=\sum_{k_{2}=1}^{\left|\mathcal{Y}_{2}\right|} p\left(x \mid \tilde{y}_{1}=k_{1}, \tilde{y}_{2}=k_{2}\right) \pi_{k_{2} \mid k_{1}}
$$

By Jensen's inequality and the concavity of mutual information we have:

$$
\begin{gathered}
\left.\sum_{k_{2}} \pi_{k_{2} \mid k_{1}} I\left(X ; Y_{1}, Y_{2} \mid \tilde{Y}_{1}=k_{1}\right)\right|_{p\left(x \mid \tilde{y}_{1}=k_{1}, \tilde{y}_{2}=k_{2}\right)} \\
\leq\left. I\left(X ; Y_{1}, Y_{2} \mid \tilde{Y}_{1}=k_{1}\right)\right|_{p\left(x \mid \tilde{y}_{1}=k_{1}\right)}
\end{gathered}
$$

To complete the proof, it remains to show that this adjustment does not change $\pi_{k_{1}}$, i.e., the marginal stationary distribution of $Y_{1}$. This fact follows from the Markov chain $Y_{1 i} \rightarrow X_{i} \rightarrow Y_{2 i}$.

We remark that the condition $p\left(y_{i} \mid x_{i}\right)>0$ in Theorem 2 can be relaxed. Even if we allow $p(y \mid x)=0$ for some $x, y$, Theorem 2 can be established following the lines in $[4$, Appendix VIII] and applying it in [5]. On the other hand, this condition holds for practical channel models, such as the binary symmetric channel with non-zero or non-one cross-over probability, and modulo additive noise channels with noise support set equal to the input alphabet.
Corollary 1 If the battery state is strongly irreducible and strongly aperiodic, the capacity with battery state information at the transmitter and receiver, $C_{S I}$, is:

$$
C_{S I}=\max _{p(x \mid \tilde{s})} \sum_{i=1}^{B} \pi_{i} I(X ; Y, S \mid \tilde{S}=i)
$$

where $\tilde{S}$ is the current battery state and $S$ is the next battery state.

We note the similarity of the capacity expression in (11) and that of Goldsmith-Varaiya expression in [11] for the capacity of fading channels with side information. Even though the channel state has input dependence, in (11) the stationary probability of the state averages out the mutual information as in Goldsmith-Varaiya expression. A recent work [12] reported a similar capacity expression for this channel with side information at both sides. We note that the expression in (11) is different from that in [12, Theorem 1]. Specifically, the expression in [12, Theorem 1] does not involve battery state as an output in the mutual information and yields lower values.

We also remark that the strongly irreducible condition is satisfied in the current energy harvesting model under some further physical conditions. In order to enable edge formation between all state pairs (see [5, Definition 2]), we need to add non-zero energy leakage probability to the battery dynamics which may or may not depend on the particular energy state. We also need that the energy arrivals can take values in the set $\left\{0,1, \ldots, E_{\max }\right\}$ with non-zero probability.

\section{A. Capacity with Side Information and Infinite-Sized Battery}

We now determine the capacity with side information and infinite-sized battery. Note that the results we have derived so far in Theorems 1 and 2 are not applicable in this case as they follow from results in [4], [5] which hold only when the cardinality of the state is finite.

Theorem 3 The capacity of the energy harvesting channel with battery state information at the transmitter and receiver and with an infinite-sized battery at the transmitter is

$$
C=\max _{p(x), E[X] \leq P_{\text {avg }}} I(X ; Y, \hat{S})
$$

where $P_{\text {avg }}$ is the average energy recharge rate $E\left[E_{i}\right]$ and the channel between $X$ and $\hat{S}$ is an additive noise channel and the noise is the energy arrival variable $E$ :

$$
\hat{S}=X-E
$$

and $\hat{S} \rightarrow X \rightarrow Y$.

Proof: The receiver can form i.i.d. realizations of the channel $p(\hat{s}, y \mid x)$ by taking the difference $\hat{S}_{i}=S_{i+1}-S_{i}$ at each channel use. Hence, the capacity of the channel $p(\hat{s}, y \mid x)$ with input constraint $E[X] \leq P_{a v g}$ is achievable by using the besteffort-transmit or save-and-transmit schemes in [1].

The converse is as follows: Let the received sequence in $n+1$ channel uses be $Y^{n+1}, S^{n+1}$ and we discard $Y_{n+1}$ which 
causes no loss of optimality as $n$ goes to infinity:

$$
\begin{aligned}
& n+1) R-H\left(W \mid Y^{n}, S^{n+1}\right)=I\left(W ; Y^{n}, S^{n+1}\right) \\
= & H\left(Y^{n}, S^{n+1}\right)-H\left(Y^{n}, S^{n+1} \mid W\right) \\
= & H\left(S_{1}\right)+\sum_{i=1}^{n} H\left(Y_{i}, S_{i+1} \mid Y^{i-1}, S^{i}\right) \\
& -\sum_{i=1}^{n} H\left(Y_{i}, S_{i+1} \mid W, Y^{i-1}, S^{i}\right) \\
\leq & \sum_{i=1}^{n} H\left(Y_{i}, S_{i+1} \mid S_{i}\right)-\sum_{i=1}^{n} H\left(Y_{i}, S_{i+1} \mid X_{i}, W, Y^{i-1}, S^{i}\right) \\
= & \sum_{i=1}^{n} H\left(Y_{i}, S_{i+1}-S_{i} \mid S_{i}\right)-\sum_{i=1}^{n} H\left(Y_{i} \mid X_{i}\right) \\
& -\sum_{i=1}^{n} H\left(S_{i+1}-S_{i} \mid X_{i}\right) \\
\leq & \sum_{i=1}^{n} H\left(Y_{i}, \hat{S}_{i}\right)-\sum_{i=1}^{n} H\left(Y_{i} \mid X_{i}\right)-\sum_{i=1}^{n} H\left(\hat{S}_{i} \mid X_{i}\right) \\
= & \sum_{i=1}^{n} I\left(X_{i} ; Y_{i}, \hat{S}_{i}\right) \\
\leq & n \max _{p(x), E[X] \leq P_{\text {avg }}} I(X ; Y, \hat{S})
\end{aligned}
$$

where (17) follows from the facts that conditioning reduces entropy and that initial battery level is finite and known to both sides and hence $H\left(S_{1}\right)=0$, (18) follows from $Y_{i} \rightarrow X_{i} \rightarrow \hat{S}_{i}$ and also from the fact that $Y_{i}, \hat{S}_{i}$ are independent of $W, Y^{i-1}, S^{i-1}$ given $X_{i}$, i.e., $W, Y^{i-1}, S^{i-1} \rightarrow$ $X_{i} \rightarrow Y_{i}, \hat{S}_{i}$. Finally, (19) follows from conditioning reduces entropy and (21) is due to the fact that the energy for the input sequence $X_{i}$ is maintained by the energy arrivals $E_{i}$ and hence $\frac{1}{n} \sum_{i=1}^{n} E\left[X_{i}\right] \leq P_{\text {avg }}$. By Fano's inequality, $H\left(W \mid Y^{n}, S^{n+1}\right)$ goes to zero as $n \rightarrow \infty$ and hence completing the proof.

Theorem 3 implies that in the infinite-sized battery case, the transmitter does not need to use the battery state information in the encoding and a single-letter code suffices to achieve the capacity. However, note that the receiver uses the battery state information to obtain the output $\hat{S}_{i}$.

\section{Achievable Schemes With Battery State INFORMATION AVAILABLE AT THE TRANSMITTER ONLY}

The capacity of the energy harvesting channel when side information is available at the transmitter only with a finitesized battery is an open problem in general [6], [7]. In this section, we identify achievable schemes for this case.

\section{A. Achievable Schemes by Shannon Strategies}

We first note that a natural achievable scheme for the energy harvesting channel with only transmitter side information is obtained by Shannon strategies [3] as emphasized in [6], [7]. The channel model in (3) fits well with the finite-state channel model with input controlled state in [8]. Let $U_{i}$ denote the Shannon strategy. For an i.i.d. $U_{i}$, we have

$$
p\left(y_{i}, u_{i}, s_{i+1} \mid s_{i}\right)=p\left(y_{i}, s_{i+1} \mid u_{i}, s_{i}\right) p\left(u_{i}\right)
$$

where

$$
p\left(y_{i}, s_{i+1} \mid u_{i}, s_{i}\right)=p\left(s_{i+1} \mid u_{i}, s_{i}\right) p\left(y_{i} \mid u_{i}\left(s_{i}\right)\right)
$$

where $p\left(y_{i} \mid u_{i}\left(s_{i}\right)\right)$ is due to the physical channel and $p\left(s_{i+1} \mid u_{i}, s_{i}\right)$ can be expressed in terms of the energy arrival process statistics and the battery size $E_{\max }$. Then, with $p\left(u_{i}\right)$ fixed, the rate $R_{p\left(u_{i}\right)}=\lim _{n \rightarrow \infty} \frac{1}{n} I\left(U^{n} ; Y^{n}\right)$ is achievable [6], [7]. The rate $R_{p\left(u_{i} \mid u_{i-1}\right)}$ can be calculated by the simulation-based method in [8]. We can then get the best achievable rate by optimizing over the probability $p\left(u_{i}\right)$. This method can be applied for a Markovian $u_{i}$ of any order [7].

\section{B. Timing-Channel Based Achievable Schemes}

Assume that the input is binary and the channel is noiseless, i.e., $p\left(y_{i} \mid x_{i}\right)=\delta\left(y_{i}-x_{i}\right)$. Moreover, energy arrival is binary with $\operatorname{Pr}\left[E_{i}=1\right]=q$. In this case, encoding and decoding can be performed over the number of channel uses between two $1 \mathrm{~s}$ and we obtain the following timing channel (see [6]):

$$
T_{n}=V_{n}+Z_{n}
$$

where $T_{n}$ is the number of channel uses between two $1 \mathrm{~s}$ in the received signal, $V_{n}$ is the number of channel uses the transmitter chooses to wait to transmit a 1 after the first energy availability, and $Z_{n}$ is the number of channel uses until the battery has at least one unit energy. The transmitter has causal information of the noise $Z_{n}$ before deciding $V_{n}$. Unlike the case with unit-sized battery as in [6], the noise process $Z_{n}$ is not i.i.d. when battery size is larger than one.

In order to fit the model to those considered in [8], we need to include, as an additional state, the available energy in the battery $B_{n}$ when $Z_{n}$ is observed. Therefore, the state of this channel is the augmented random variables $\left(Z_{n}, B_{n}\right)$. Let $U_{n}$ denote an auxiliary i.i.d. random sequence. Then, we have:

$$
\begin{aligned}
& p\left(z_{n+1}, b_{n+1}, t_{n}, u_{n} \mid z_{n}, b_{n}\right) \\
& \quad=\delta\left(t_{n}-f\left(u_{n}, z_{n}, b_{n}\right)\right) p\left(z_{n+1}, b_{n+1}, u_{n} \mid z_{n}, b_{n}\right)
\end{aligned}
$$

where $f\left(u_{n}, z_{n}, b_{n}\right)$ is a function that determines the Shannon strategy. Moreover, we have:

$$
p\left(z_{n+1}, b_{n+1}, u_{n} \mid z_{n}, b_{n}\right)=p\left(z_{n+1}, b_{n+1} \mid u_{n}, z_{n}, b_{n}\right) p\left(u_{n}\right)
$$

Here, $p\left(z_{n+1}, b_{n+1} \mid u_{n}, z_{n}, b_{n}\right)$ is determined by the energy arrival process statistics only. Specifically, if energy arrives during the waiting time $V_{n}=f\left(U_{n}, Z_{n}, B_{n}\right)$, next noise level is $Z_{n+1}=0$ and $B_{n+1}$ is found depending on the battery size.

In view of (25), the following rate is achievable and it can be evaluated by the method in [8]:

$$
R=\lim _{n \rightarrow \infty} \frac{\frac{1}{n} I\left(U^{n} ; T^{n}\right)}{\frac{1}{n} \sum_{i=1}^{n} E\left[T_{i}\right]}
$$




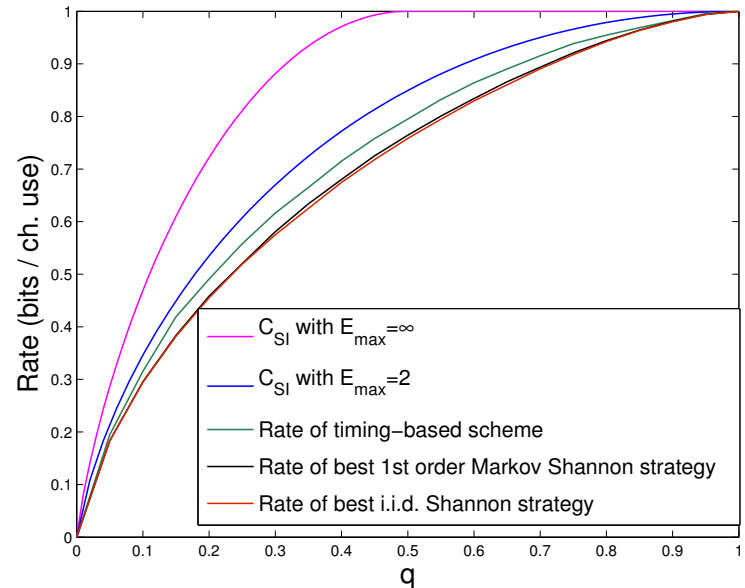

Fig. 3. The capacity with battery state information at the receiver side and achievable rates with side information at the transmitter only in the noiseless binary channel. The plot is with respect to the energy arrival probability $q$.

We note that this achievable scheme is possibly sub-optimal as it does not update the strategy after the observation of a new energy arrival allocated in the battery. This constitutes a possible direction for improving the achievable scheme.

\section{NumericAl RESUlts}

In this section, we evaluate the capacity and achievable rates with and without receiver side information. In the timing-based achievable scheme, we use an extended version of the auxiliary selection in [6] as follows. Let $U \in\{0,1, \ldots, N-1\}$,

$$
V= \begin{cases}U-Z+1, & U \geq Z \\ (U-Z \bmod M)+1, & U<Z\end{cases}
$$

where $M<N$. Note that this particular scheme does not use $B_{n}$ information available at the transmitter. We choose $M$ and $p(u)$ in the simulation and calculate the achievable rate.

In Fig. 3, we plot the achievable rates by the best i.i.d. [6], [7] and the best first order Markovian [7] Shannon strategies and the timing-based achievable scheme when the channel is noiseless binary, $E_{\max }=2$ and the battery state information is available only at the transmitter. We also plot the capacity with battery state information at both sides for $E_{\max }=2$ and $E_{\max }=\infty$. Note that when $E_{\max }=\infty$, the availability of the battery state information at the receiver does not increase the capacity for the noiseless channel. We observe that the timing-based achievable scheme performs better than zeroth and first order Markovian Shannon strategies.

In Fig. 4, we plot the achievable rates and the capacity without and with receiver side information, respectively, in a binary symmetric channel with crossover probability $p_{e}$ for $E_{\max }=2$ and $q=0.5$ with respect to $p_{e}$. We also plot the capacity for $E_{\max }=\infty$.

\section{CONCLUSION}

We determined the capacity of an energy harvesting channel with an energy harvesting transmitter and battery state information available at the transmitter and receiver sides. This is an instance of a finite-state channel and the channel

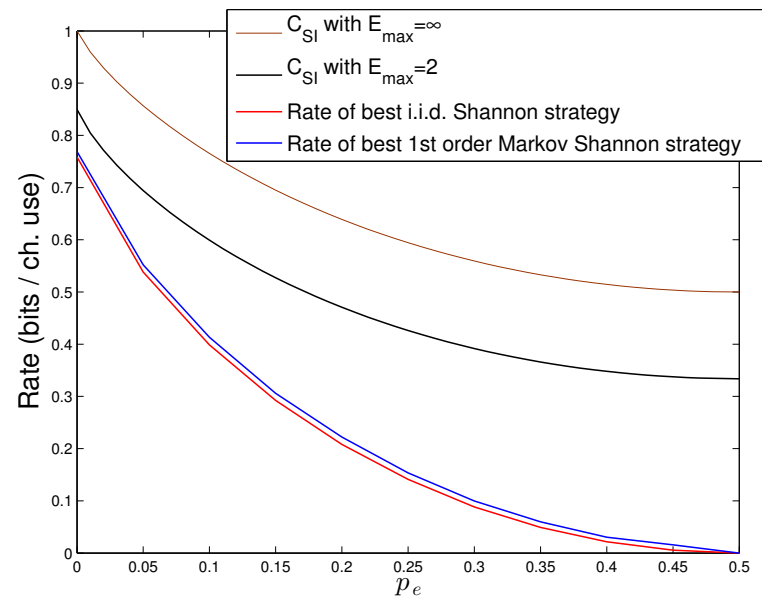

Fig. 4. The capacity with battery side information at the receiver side and achievable rates with side information only at the transmitter in a $\mathrm{BSC}\left(p_{e}\right)$. The plot is with respect to the channel crossover probability $p_{e}$ for $q=0.5$.

output feedback does not increase the capacity. We stated the capacity as maximum directed mutual information from the input to the output and the battery state. We identified sufficient conditions for the channel to have stationary input distributions as optimal distributions. We also derived a singleletter capacity expression for this channel with battery state information at both sides and infinite-sized battery at the transmitter. Next, we extended the achievable scheme in [6] to a noiseless channel with $E_{\max }>1$. We provided a simulationbased method to evaluate the achievable rates using [8].

\section{REFERENCES}

[1] O. Ozel and S. Ulukus. Achieving AWGN capacity under stochastic energy harvesting. IEEE Trans. on Inform. Theory, 58(10):6471-6483, Oct. 2012

[2] O. Ozel and S. Ulukus. AWGN channel under time-varying amplitude constraints with causal information at the transmitter. In Asilomar Conf., Nov. 2011.

[3] C. E. Shannon. Channels with side information at the transmitter. IBM journal of Research and Development, 2(4):289-293, 1958.

[4] H. Permuter, T. Weissman, and A. Goldsmith. Finite state channels with time-invariant deterministic feedback. IEEE Trans. on Inform. Theory, 55(2):644-662, February 2009.

[5] J. Chen and T. Berger. The capacity of finite-state Markov channels with feedback. IEEE Trans. on Inform. Theory, 51(3):780-798, March 2005.

[6] K. Tutuncuoglu, O. Ozel, A. Yener, and S. Ulukus. Binary energy harvesting channel with finite energy storage. In IEEE ISIT, July 2013.

[7] W. Mao and B. Hassibi. On the capacity of a communication system with energy harvesting and a limited battery. In IEEE ISIT, July 2013.

[8] D. M. Arnold, H.-A. Loeliger, P. O. Vontobel, A. Kavcic, and W. Zeng. Simulation-based computation of information rates for channels with memory. IEEE Trans. on Inform. Theory, 52(8):3498-3508, August 2006.

[9] I. Naiss and H. Permuter. Extension of the Blahut-Arimoto algorithm for maximizing directed information. IEEE Trans. on Inform. Theory, 59(1):204-223, January 2013.

[10] S. Tatikonda and S. Mitter. Capacity of channels with feedback. IEEE Trans. on Inform. Theory, 55(1):323-349, January 2009.

[11] A. Goldsmith and P. Varaiya. Capacity of fading channels with channel side information. IEEE Trans. on Inform. Theory, 43(6):1986-1992, November 1997.

[12] P.K. Deekshith, V. Sharma, and R. Rajesh. AWGN channel capacity of energy harvesting transmitters with a finite energy buffer. Available at arXiv:1307.4505. 